\title{
Multiple Internal Resonances in MEMS Arch Resonators
}

\author{
A.Z. Hajjaj, N. Jaber, M.A.A. Hafiz, S. Ilyas, and M.I. Younis ${ }^{a}$ \\ Physical Science and Engineering Division, King Abdullah University of Science and Technology, Thuwal, 23955- \\ 6900, Saudi Arabia.
}

\begin{abstract}
Micromachined shallow arch resonant beams have attracted significant attention thanks to their rich dynamical behavior, inherent nonlinearities, and the potential to excite various internal resonances. Currently, there is a lack of comprehensive experimental studies for the various types of internal resonances in arches and particularly at the micro and nano scales. Here, we aim to investigate and identify different types of internal resonances of an initially curved beam, electrothermally actuated and electrostatically driven, by electrical characterization techniques. Upon changing the electrothermal voltage of silicon micromachined arches, the second symmetric natural frequency of an arch is adjusted to near twice, three times, and four times the fundamental natural frequency, which gives rise to 2:1, 3:1, and 4:1 autoparametric resonances between the two modes. These resonances are demonstrated experimentally. We show various frequency-response curves of the total response around the excitation frequency and highlight the contribution of each mode before, during, and after the internal resonances. Allandeviation results are also shown indicating enhanced frequency stabilization of the arch oscillation when experiencing internal resonances. These studies motivate further research in this direction to exploit internal resonances of micromachined resonators for practical applications, such as sensors and mechanical amplifier.
\end{abstract}

Keywords: Micromachined arch resonator, nonlinearity, internal resonance, electrothermal and electrostatic actuation

\section{Introduction}

Nano/microelectromechanical systems (N/MEMS) have demonstrated exciting capabilities and superior features for several applications, such as mass/gas sensing [1-2], energy harvesting [3-4], logic [5-6] filtering [7-8], and communications [9]. On a fundamental level, N/MEMS have enabled in-depth studies for various physical phenomena thanks to the ability to precisely and easily control various physical parameters, such as damping, stiffness, and boundary conditions. Particularly, the nonlinear interactions among different vibrational modes of N/MEMS resonators have been the focus of intense research in the past few decades [8-19]. These modes can be coupled nonlinearly among the structure itself or through internal resonances.

Bistable structures, such as arch beams, have attracted significant attention thanks to their rich static (snap-through, jumps, pull-in) and dynamical behaviors. Arch beams have inherent nonlinearities (cubic from stretching and quadratic from initial curvature, as viewed in the system equation of motion), which add complexity to their dynamics. These structures have been

${ }^{\text {a }}$ Corresponding author: mohammad.younis@ @aust.edu.sa 
shown recently to exhibit various internal (autoparametric) resonances among different vibrational modes $(1: 1,2: 1,3: 1)[19$ 20]. These internal resonances have been proposed for various practical applications, such as frequency stabilization [9, 12], energy dissipation [10], and energy harvesting [3-4]. Hence, understanding the dynamics of these structures during internal resonance is crucial for the successful implementation of these devices.

The overall response of an arch beam electrothermally tuned and electrostatically activated was investigated experimentally, using optical techniques, as experiencing 2:1 and 3:1 internal resonance [19]. Recently [9-10], internal resonance has been proposed to improve the frequency stabilization of self-sustained resonators as well as to compensate the energy losses. This approach was demonstrated for a 3:1 internal resonance between the $1^{\text {st }}$ bending and torsional modes.

Based on electrical characterization techniques, this work aims to investigate different types of internal resonances of an initially curved beam and to characterize in-depth the dynamics during these internal resonances.

\section{Experimental Setup}

The experiments were conducted on electrothermally tuned and electrostatically driven clamped-clamped arch MEMS resonators. These arch beams are fabricated on a highly conductive $30 \mu \mathrm{m}$ Si device layer of a silicon-on-insulator (SOI) wafer by a two-mask process using standard photo-lithography, metal sputtering, deep reactive ion etch (DRIE), and vapor hydrofluoric acid dry (vpour-HF) etch [5]. Here the arch beam has a length of $700 \mu \mathrm{m}$, a depth of $30 \mu \mathrm{m}$, a width of $2 \mu \mathrm{m}$, and an initial rise $2.6 \mu \mathrm{m}$. The arch beam is biased with a DC voltage source that was fixed at $65 \mathrm{~V}$ for all the experiments. The drive electrode provides an AC harmonic voltage. A low-noise amplifier (LNA) is used to amplify the output AC motional current induced at the sense electrode due to the microbeam in-plane motion. Then, the motional output current is simultaneously measured using a network analyzer (Agilent 5071C) and spectrum analyzer. The network analyzer records the total response of the resonator while the spectrum analyzer shows the contribution of the other activated mode in the total response, Fig. 1(a). All the experiments are conducted at room temperature and in vacuum (pressure 6 mTor).

To control the stiffness of the arch beam, and hence its resonance frequency, a separate DC voltage source, $V_{T h}$, is connected to the arch beam anchors to induce a constant current flowing through the beam, and hence heating it up by Joule's heating. Figure 1(b) shows the measured first two symmetric resonance frequencies of the arch beam. Upon changing the electrothermal voltage, the first resonance frequency increases up to twice its fundamental value $(71 \mathrm{kHz})$; due to the continuous increase in the arch curvature (stiffness) [8]; and the third resonance frequency decreases, Fig. 1(b). Hence the ratios of 2:1 and 3:1 between the two modes can be achieved as varying $V_{T h}$, as shown in the inset of Fig. 1(b), which presents a necessary condition to activate the corresponding internal resonances. 


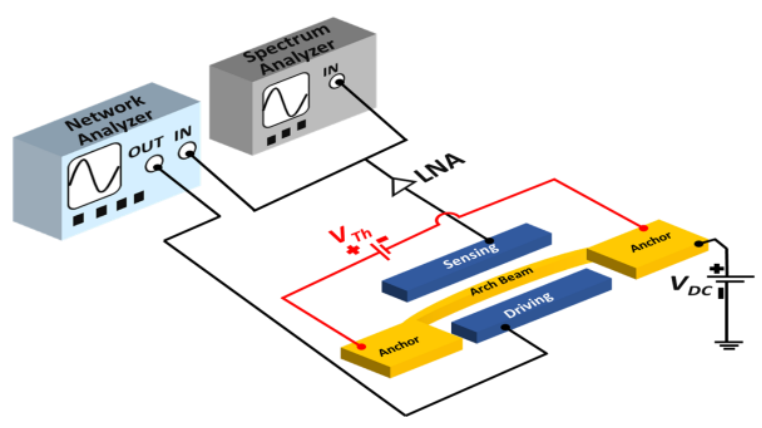

(a)

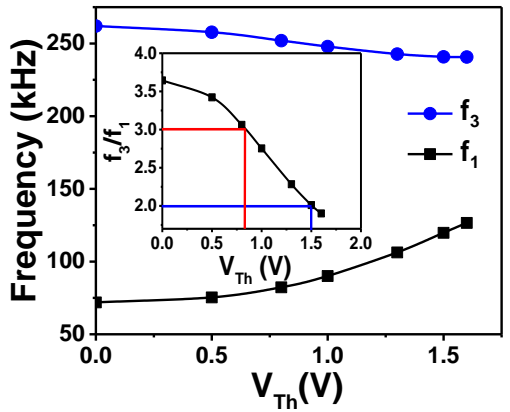

(b)

Fig.1. (a) A schematic of the experimental setup. (b) The variation of the first two symmetric frequencies of the arch beam; $700 \mu \mathrm{m}$ length, $30 \mu \mathrm{m}$ depth, and $2 \mu \mathrm{m}$ width; with the electrothermal voltage $V_{T h}$. The arch beam has a $2.6 \mu \mathrm{m}$ initial rise (distance between its midpoint to its straight beam level). The inset shows the variation of the ratio between the $3^{\text {rd }}$ and $1^{\text {st }}$ mode frequencies with $V_{T h}$.

\section{Results}

\section{A. 2:1 internal Resonance}

As fixing $V_{T h}$ at a ratio of two $\left(V_{T h}=1.509 \mathrm{~V}\right)$, we sweep the excitation frequency around the $1^{\text {st }}$ mode (respectively the $\left.3^{\text {rd }}\right)$. Meanwhile, the output signal spectrum is examined, using the spectrum analyzer, in the vicinity of the $3^{\text {rd }}$ mode (respectively the $1^{\text {st}}$ ) to identify its contribution on the overall response showen by the network analyzer, Fig. 2 (respectively Fig. 3). The figures indicate a frequency component around the $3^{\text {rd }}$ mode (respectively the $1^{\text {st }}$ ). Figure $2($ a) shows that even for small AC excitation voltage the response of the beam is dominated by the cubic nonlinearities showing a hardening response. This illustrates the strong interaction between both modes since the natural response of the arch beam around the $1^{\text {st }}$ resonance frequency is dominated by the quadratic nonlinearity (softening effect) [19-20]. Increasing the AC bias voltage gradually results in the splitting of the overall vibration amplitude into two peaks where the arch beam is experiencing internal resonance. In Figs. 2(b)-(d) (respectively Figs. 3(a)-(c) ), the presence of two peaks suggests a nonlinear modal interaction among the $1^{\text {st }}$ and $3^{\text {rd }}$ resonance frequencies. This proves the transfer of energy from the first mode to the third mode and vice versa. This energy transfer can be shown by the detected current around the $3^{\text {rd }}$ resonance frequency (respectively $1^{\text {st }}$ frequency) while exciting the $1^{\text {st }}$ resonance frequency (respectively the $3^{\text {rd }}$ frequency).

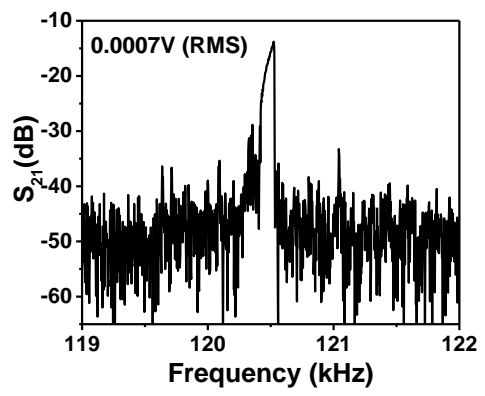

(a) 


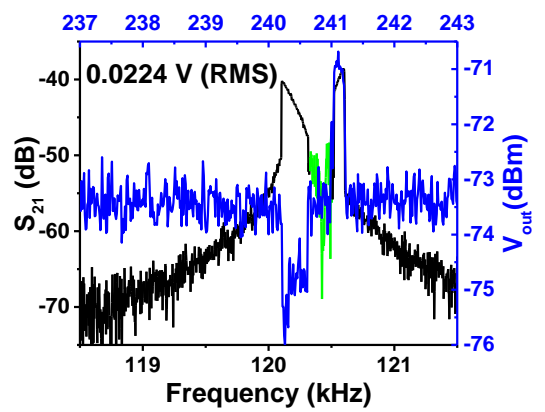

(b)

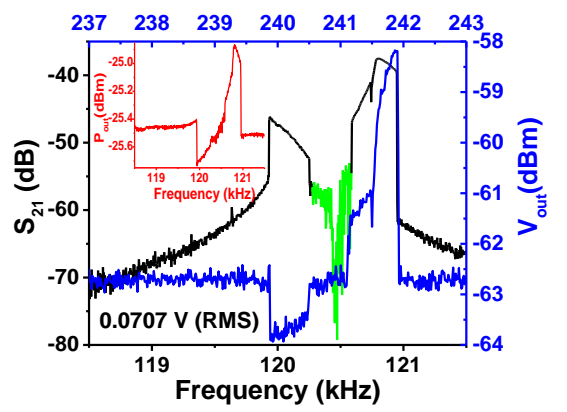

(c)

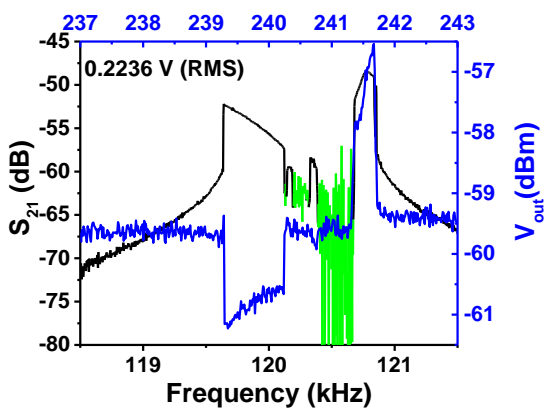

(d)

Fig.2. (a), (b), (c) and (d) The frequency response of the arch beam around the $1^{\text {st }}$ resonance frequency for various AC harmonic excitations showing the 2:1 internal resonance, at $V_{T h}=1.509 \mathrm{~V}$. The inset in (b) shows the contribution of the $1^{\text {st }}$ mode on the overall response.

Then, as applying larger driving voltages while sweeping around the $1^{\text {st }}$ mode, the arch beam passes from stable to unstable branches showing a possible quasi-periodic motion (the green regions) which could be a consequence of a reverse Hopf bifurcation, Figs. 2(b)-(d). The quasi-periodic motion presents a common characteristic of the internal resonance suggesting the immersion of a finite number of incommensurate frequencies on the dynamic response of the arch beam [17]. Driving the system more strongly enhances the coexistence of multi-stable states for larger bandwidth and hence might lead to more complicated dynamic phenomena such as chaotic responses.

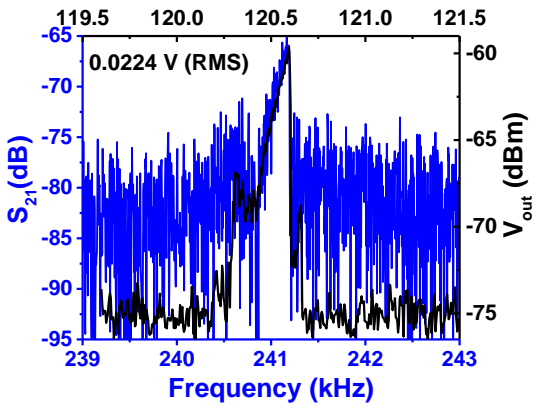

(a)

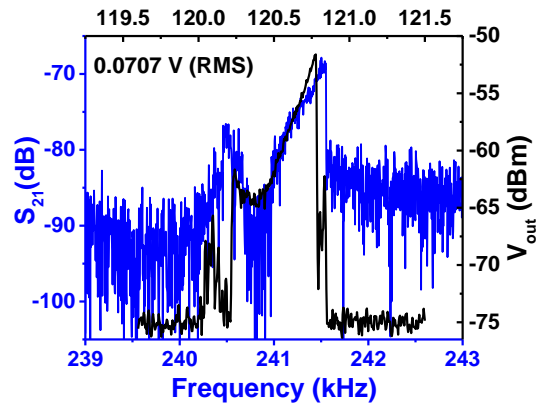

(b)

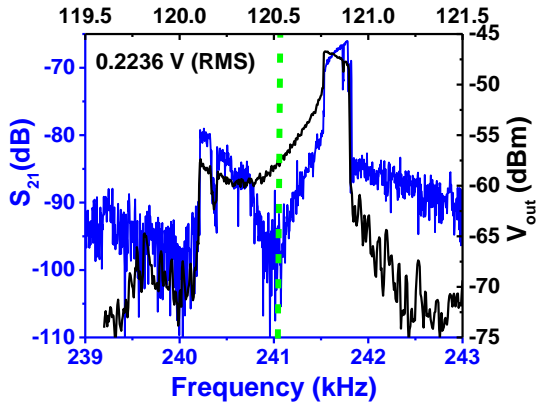

(c)

Fig.3. (a), (b), and (c) The frequency response of the arch beam around the $3^{\text {rd }}$ frequency for various AC harmonic excitations showing the $2: 1$ internal resonance, at $V_{T h}=1.509 \mathrm{~V}$.

Figure 3 shows that while sweeping around the $3^{\text {rd }}$ mode, the arch beam does not experience any unstable motion. However, Fig. 3(c) shows that even when if no oscillation is detected around the $3^{\text {rd }}$ mode (dashed line section), a current is sensed around the $1^{\text {st }}$ mode that acts as a reservoir of the energy of the higher mode at the steady state.

\section{B. $3: 1$ and $4: 1$ internal Resonance}

Next, we tune $V_{T h}$ to a ratio three $\left(V_{T h}=0.845 \mathrm{~V}\right)$. As sweeping the frequency around the first mode, the $1^{\text {st }}$ and $3^{\text {rd }}$ frequencies start to interact leading to sharp dips in frequency that could be explained by the falling to a lower stable branch 
due to the transfer of the energy to the $3^{\text {rd }}$ mode, Figs. 4(a)-(c). The figures show that the quadratic nonlinearity dominates the response despite the mode interaction. The amplitude of the $3^{\text {rd }}$ mode contribution appears to have a hardening behavior (due to the cubic nonlinearity), which is normal nonlinear response of an arch beam around the $3^{\text {rd }}$ mode $[20,22]$.

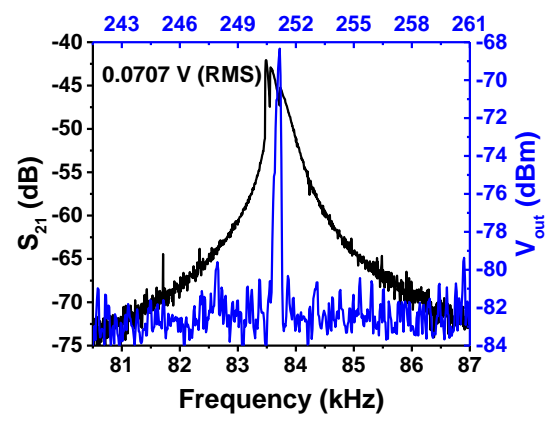

(a)

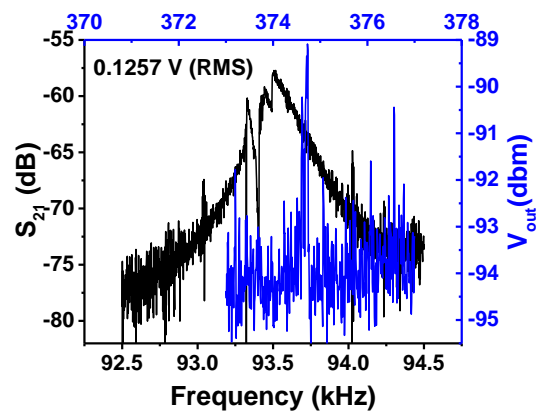

(d)

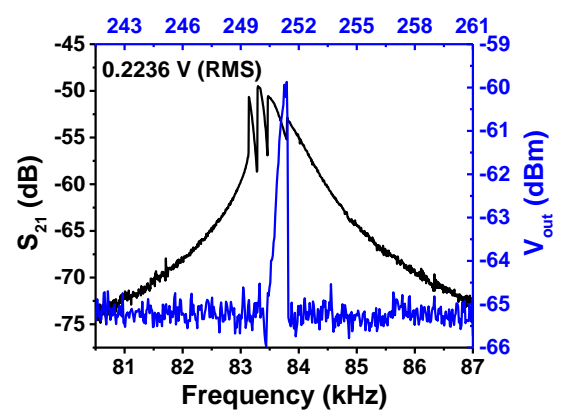

(b)

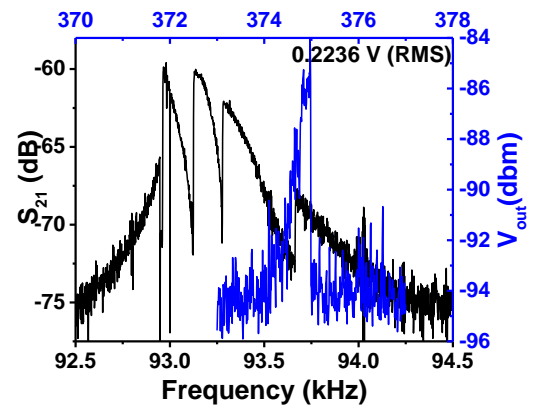

(e)

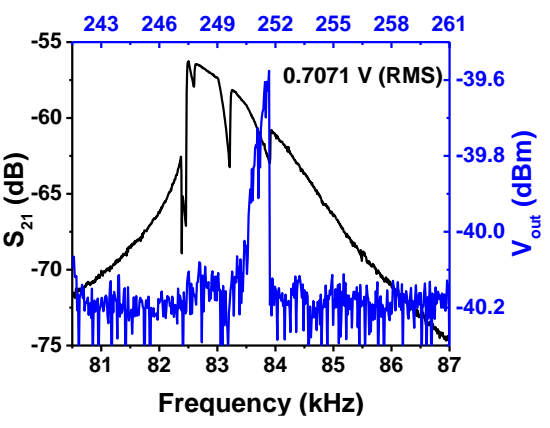

(c)

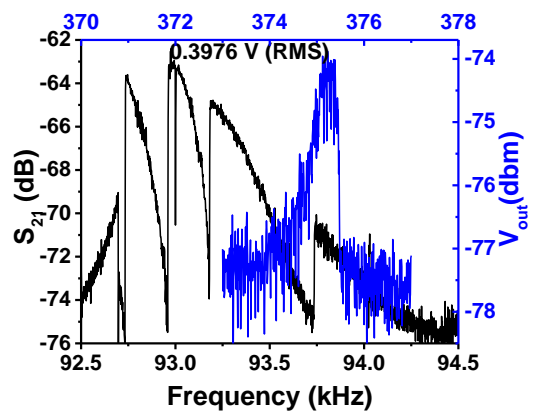

(f)

Fig.4. (a), (b), and (c) The frequency response of the arch beam around the $1^{\text {st }}$ frequency for various AC harmonic excitations showing the 3:1 internal resonance, at $V_{T h}=0.845 \mathrm{~V}$. (d), (e), and (f) The frequency response of the arch beam around the $1^{\text {st }}$ frequency for various AC harmonic excitations showing the 4:1 internal resonance, at $V_{T h}=0.76 \mathrm{~V}$.

For a similar arch beam with length $650 \mu \mathrm{m}$ and a thickness of $3 \mu \mathrm{m}$, a ratio $4: 1$ between the $1^{\text {st }}$ and $3^{\text {rd }}$ modes was achieved. As known in the literature that the $4^{\text {th }}$ order coupling between the $1^{\text {st }}$ and $3^{\text {rd }}$ resonance frequencies is weak compared to other couplings, hence, can be thought as negligible. However, in this work, we show that the 4:1 internal resonance could be activated by driving the arch beam highly at vacuum, Figs. 4(d)-(f). This interaction is attributed to a quartic nonlinearity, which can be from the higher order nonlinearity geometric nonlinearity or that from the electrostatic force. Also, one should mention here that the qualitative response of the 4:1 internal resonance appears similar to the 3:1 internal resonance.

\section{Theory}

To model the nonlinear behavior of the arch resonator, we refer to the Euler Bernoulli beam theory. The transverse vibration of the arch beam of an initial shape $w_{0}(x)$ under double-side electrostatic actuation is governed by [19]: 


$$
\begin{aligned}
& \rho b h \frac{\partial^{2} w}{\partial t^{2}}+c \frac{\partial w}{\partial t}+E I \frac{\partial^{4} w}{\partial x^{4}}=\underbrace{\left(\frac{\partial^{2} w}{\partial x^{2}}+\frac{d^{2} w_{0}}{d x^{2}}\right)\left[N+\frac{E A}{2 l} \int_{0}^{l}\left(\left(\frac{\partial w}{\partial x}\right)^{2}+2 \frac{\partial w}{\partial x} \frac{d w_{0}}{d x}\right) d x\right]}_{\text {Curvature (quadratic) }+ \text { Midplane Stretching (cubic) }}
\end{aligned}
$$

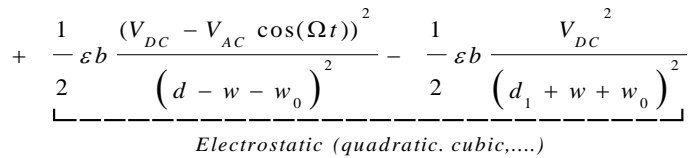

The arch beam is subjected to the clamped-clamped boundary conditions as

$$
w(0, t)=w(l, t)=0 \text { and }\left.\frac{\partial w}{\partial x}\right|_{(0, t)}=\left.\frac{\partial w}{\partial x}\right|_{(l, t)}=0
$$

In Eq. (1), $x$ is the position along the arch beam, $t$ is time, $l, b$ and $h$, are, respectively, the length, width, and thickness of the arch beam, $\rho$ is the material density, $I$ is the moment of inertia of the rectangular cross-sectional area $A=b h$, and $E$ is Young's modulus. The term $N$ represents the total axial load on the structure accounting for the residual load arising from the fabrication process, and the thermal compressive load induced by the applied $V_{T h}$. The last two terms in Eq. (1) represent the electrostatic force when the two-port measurement is used, where $V_{D C}$ is the DC polarization voltage and $V_{A C}$ is the AC harmonic voltage of frequency $\Omega$. The arch resonator is separated from the driving/sensing electrodes with gap widths $d$ and $d_{l}$, respectively. The beam is subjected to a viscous damping of coefficient $c$.

According to Eq. (1), the response of the arch is governed by two types of nonlinearity: quadratic and cubic nonlinearities. The quadratic nonlinearity is originated from the arch curvature and the electrostatic force. The cubic nonlinearity is a geometric nonlinearity originated from the midplane stretching. Next, using a three-mode Galerkin reduced-order model [19], the frequency response of the arch beam is simulated for different AC excitations for a case of 2:1, Fig. 5, and 3:1 Fig. 6 internal resonances.

As the arch starting to experience the 2:1 internal resonance, the frequency response is split into two peaks, Figs. 5 (a)-(b). Increasing the forcing further, Figs. 5(c)-(d), the arch response passes through a quasi-periodic motion, as evident in the Poincare section in the insets of the figures, which can be a consequence of a Hopf bifurcation that is common in such cases [20]. Evidences of complex (potentially chaotic) behavior can be seen in Fig. $5 \mathrm{~d}$.

For the 3:1 internal resonance, a higher excitation $\mathrm{AC}$ is needed to activate the nonlinear interaction between the contributing modes. Fig. 6 shows the simulated results for the cases of backward and forward frequency sweeps. As experiencing internal resonance, Fig. 6a shows a simple softening behavior, which is then interrupted by jumps as AC is further increased, Fig. 6b, Fig. 6c. Fig. 6c also shows signs of complex behavior. 


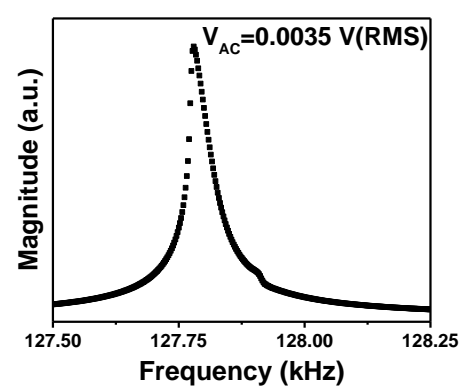

(a)

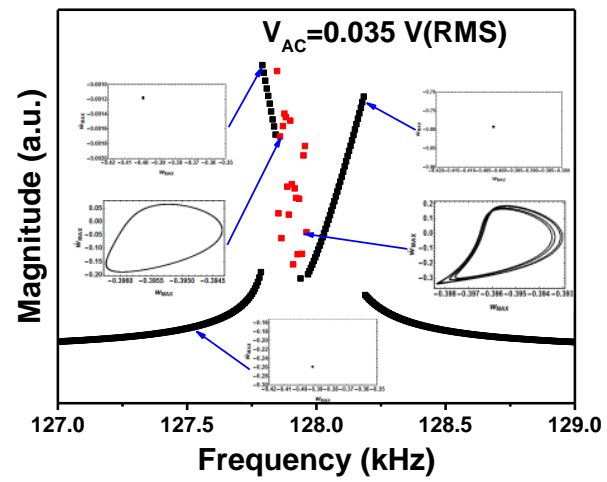

(c)

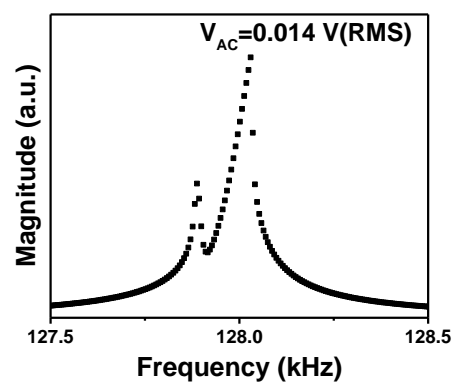

(b)

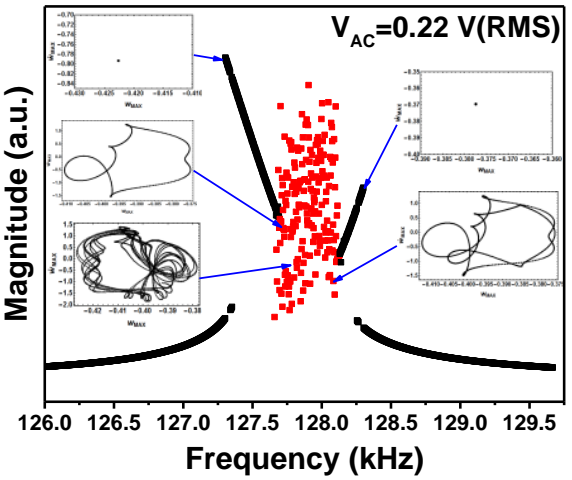

(d)

Fig. 5. Frequency response curves of the arch beam around the $1^{\text {st }}$ resonance frequency for various AC harmonic excitations near the 2:1 internal resonance. $V_{D C}$ is fixed at $65 \mathrm{~V}$. The assumed damping ratio is 0.00013 . The red scattered data show the quasi-periodic motion of the arch. Insets show Poincaré sections of selected points.

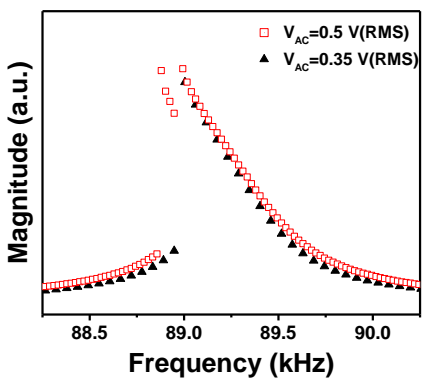

(a)

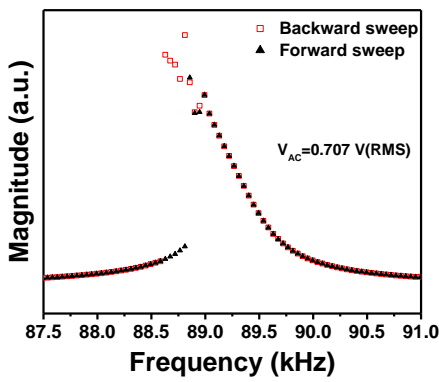

(b)

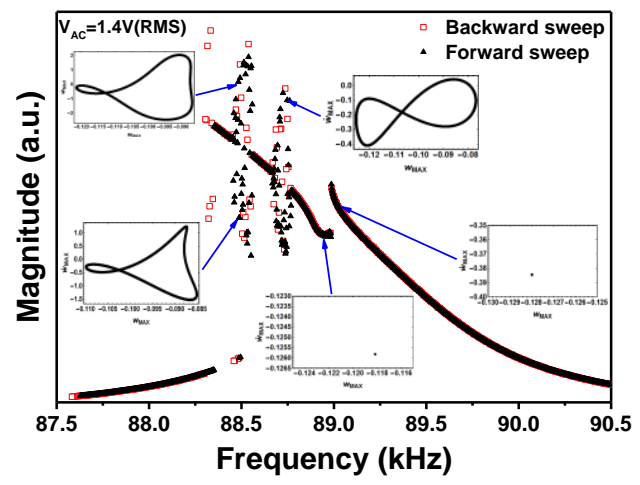

(c) 
Fig. 6. The frequency response of the arch beam around the $1^{\text {st }}$ resonance frequency showing the 3:1 internal resonance for different AC excitation. $V_{D C}$ is fixed at $65 \mathrm{~V}$. The assumed damping ratio is 0.00018 . Insets in (c) show Poincaré sections of selected points.

\section{Discussion}

Moreover, one should note that the arch response is highly dependent on the surrounding pressure [21]. Pressure variation leads to a different operating $V_{T h}$ to activate different internal resonances. Also, increasing the surrounding pressure leads to the increase of the viscous friction coefficient (i.e. viscous damping coefficient $c$ ) in the equation of motion governing the arch vibration, Eq. (1). Consequently, the pressure variation affects the interaction region and bandwidths. Figure 7(a) shows that as increasing the pressure, the bandwidth of the unstable motion shrinks down with conserving the same interaction behavior, at the same excitation voltage. However, as increasing the pressure, the dip of the frequency response induced by the $3: 1$ internal resonance shown previously decreases until disappearing at a relatively higher pressure, Fig. 7(b). Hence, at a relatively higher pressure, the response is dominated by the quadratic nonlinearity and then passes by the internal resonance without falling to the other lower branch (frequency dip) [22].

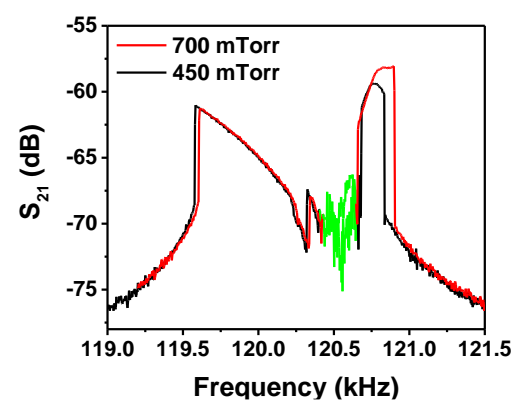

(a)

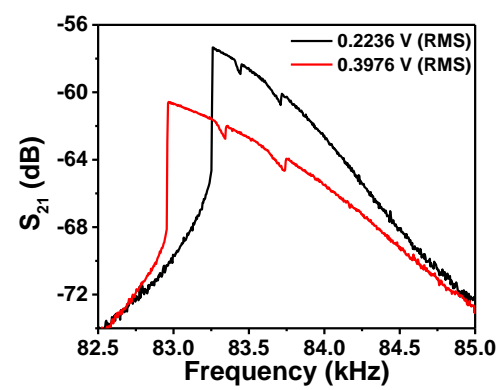

(b)

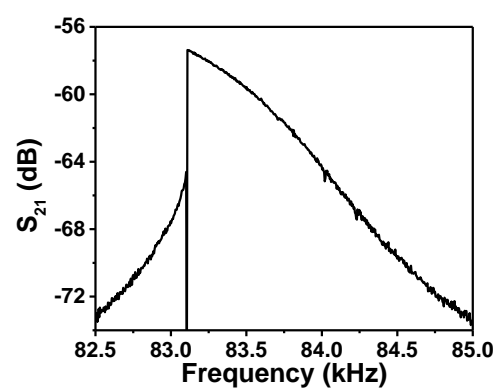

(c)

Fig.7. (a) The frequency response as experiencing the $2: 1$ internal resonance for $0.2236 \mathrm{~V}$ (RMS) excitation voltage for different surrounding pressure. At $450 \mathrm{mTorr}$ pressure, the applied $V_{T h}$ is equal to $1.521 \mathrm{~V}$. At $700 \mathrm{mTorr}$ pressure, the applied $V_{T h}$ is equal to $1.53 \mathrm{~V}$. (b) The frequency response as experiencing the 3:1 internal resonance at $450 \mathrm{mTorr}$ and for different AC harmonic excitations, at $V_{T h}=0.86$.

(c) The frequency response as experiencing the 3:1 internal resonance at $700 \mathrm{mTorr}$ and for $0.2236 \mathrm{~V}$ (RMS) excitation voltage, at $V_{T h}=$ 0.875 .

Next, we investigate and characterize the effect of different internal resonances $(2: 1$ and $3: 1)$ on the frequency stabilization of the studied system. Hence, a frequency noise analysis can be obtained by analyzing the Allan deviation for different cases: outside and inside internal resonance region. The effect of the noise on the resonator oscillation is given by the average of the fractional frequency fluctuation over an interval $\tau$, as a function of that averaging time $\tau$. Then, the Allan deviation [23-24] $\sigma_{y}$ ( $\tau)$ can be expressed as:

$\sigma_{y}(\tau)=\sqrt{\frac{1}{2(N-1)}\left(\sum_{i=1}^{N-1}\left(\bar{f}_{i+1}^{\tau}-\bar{f}_{i}^{\tau}\right)^{2}\right)}$ 
where $\bar{f}_{i}^{\tau}$ denotes the relative frequency fluctuation averaged over the $i^{\text {th }}$ discrete time interval of $\tau$ defined as

$$
\bar{f}_{i}(\tau)=\frac{f(i)-f_{0}}{f_{0}}
$$

where $f_{0}$ is the driving frequency. Figure 8 shows the Allan deviation of the arch resonator, in an open-loop configuration, at a constant AC voltage (0.22 V RMS) outside and within the internal resonance regime. The electrothermal voltage is tuned off to zero to study the frequency stabilization away from any internal resonance activation. The measurements are conducted by monitoring the amplitude fluctuation for a specified period of time, at the constant $f_{0}$. The Allan deviation curves seem to have a standard curve behavior as expected in a MEMS oscillator. At low integration time, the noise is dominated by white noise while at higher integration time the fluctuation is governed by the thermal drift.

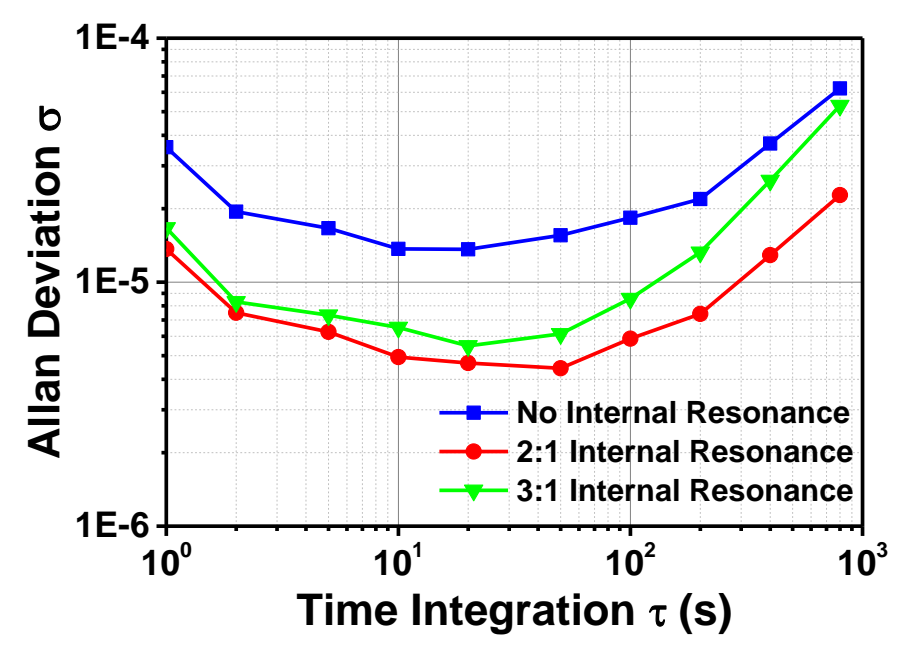

Fig. 8. Allan deviation of the arch resonator showing the improvement of frequency stabilization inside the internal resonance regime. The driving power is fixed at $0.22 \mathrm{~V}(\mathrm{RMS})$.

Figure 8 demonstrates the one-order-of-magnitude enhanced stability of the resonator response inside the internal resonance regime. Also, the frequency fluctuation around the driving frequency decreases from $\pm 1 \mathrm{~Hz}$ to $\pm 0.45 \mathrm{~Hz}$ as operating in the internal resonance regions. Both internal resonance types (2:1 and 3:1) show almost similar improvement on the stability of the resonator response. At low pressure, operating the arch beam in the 3:1 internal resonance interaction might lead to a safe operation since it gives the same frequency stability and it prevents the oscillation from the unstable branches due to the Hopf bifurcations. One should mention here that these results can be improved by using a closed-loop configuration. The activated internal resonances are independent of the arch size. Hence, the proposed mechanism can be used to enhance the stability of nanoscale resonator. 


\section{Conclusions}

In conclusion, we investigated the nonlinear mode coupling between the first two symmetric modes of an initially curved MEMS resonators via different types of internal resonances. The curved beam is electrothermally tuned and electrostatically driven. While maintaining a constant electrothermal voltage, the stiffness, and hence the frequencies, of the arch beam is monitored, and different commensurate ratios among various modes could be attained. As dynamically driving the arch beam at a fixed ratio, the storing of the energy of the contributing mode was demonstrated for different types of internal resonances. As driving the arch resonator in the interaction regime, an improvement of the frequency stabilization is demonstrated. These studies motivate further research in this direction to exploit internal resonances of arch resonators for practical applications, such as sensors and mechanical amplifier.

\section{Acknowledgment:}

This research has been supported through King Abdullah University of Science and Technology (KAUST) fund.

\section{References}

[1] N. Kacem, J. Arcamone, F. Perez-Murano, S. Hentz, Journal of Micromechanics and Microengineering, 20 (2010) 045023.

[2] N. Jaber, S. Ilyas, O. Shekhah, M. Eddaoudi, M.I. Younis, Journal of Microelectromechanical Systems, (2018).

[3] L. Xiong, L. Tang, B.R. Mace, Applied Physics Letters, 108 (2016) 203901.

[4] C. Lan, W. Qin, W. Deng, Applied Physics Letters, 107 (2015) 093902.

[5] M.A.A. Hafiz, L. Kosuru, M.I. Younis, Nature communications, 7 (2016) 11137.

[6] A. Yao, T. Hikihara, Physics Letters A, 377 (2013) 2551-2555.

[7] M.A. Al Hafiz, L. Kosuru, A.Z. Hajjaj, M.I. Younis, IEEE Transactions on Electron Devices, 64 (2017) 3392-3398.

[8] A.Z. Hajjaj, M.A. Hafiz, M.I. Younis, Scientific Reports, 7 (2017) 41820.

[9] D. Antonio, D.H. Zanette, D. López, Nature communications, 3 (2012) 806.

[10] C. Chen, D.H. Zanette, D.A. Czaplewski, S. Shaw, D. López, Nature communications, 8 (2017) 15523.

[11] I. Mahboob, R. Dupuy, K. Nishiguchi, A. Fujiwara, H. Yamaguchi, Applied Physics Letters, 109 (2016) 073101.

[12] D. Pu, X. Wei, L. Xu, Z. Jiang, R. Huan, Applied Physics Letters, 112 (2018) 013503.

[13] H. Okamoto, R. Schilling, H. Schütz, V. Sudhir, D.J. Wilson, H. Yamaguchi, T.J. Kippenberg, Applied Physics Letters, 108 (2016) 153105.

[14] I. Mahboob, V. Nier, K. Nishiguchi, A. Fujiwara, H. Yamaguchi, Applied Physics Letters, 103 (2013) 153105.

[15] G. Verbiest, D. Xu, M. Goldsche, T. Khodkov, S. Barzanjeh, N. von den Driesch, D. Buca, C. Stampfer, Applied Physics Letters, 109 (2016) 143507.

[16] A. Ganesan, A. Seshia, Applied Physics Letters, 112 (2018) 084102.

[17] W.J. Venstra, R. van Leeuwen, H.S. van der Zant, Applied Physics Letters, 101 (2012) 243111.

[18] C. Samanta, P. Yasasvi Gangavarapu, A. Naik, Applied Physics Letters, 107 (2015) 173110.

[19] A.H. Ramini, A.Z. Hajjaj, M.I. Younis, Scientific reports, 6 (2016) 34717.

[20] A.H. Nayfeh, Nonlinear interactions: analytical, computational and experimental methods, Wiley, 2000. 
[21] A.Z. Hajjaj, N. Alcheikh, M.A.A. Hafiz, S. Ilyas, M.I. Younis, Applied Physics Letters, 111 (2017) 223503.

[22] H.M. Ouakad, H.M. Sedighi, M.I. Younis, Journal of Computational and Nonlinear Dynamics, 12 (2017) 051025.

[23] D.W. Allan, IEEE transactions on ultrasonics, ferroelectrics, and frequency control, 34 (1987) 647-654.

[24] M. Sansa, E. Sage, E.C. Bullard, M. Gély, T. Alava, E. Colinet, A.K. Naik, L.G. Villanueva, L. Duraffourg, M.L. Roukes, Nature nanotechnology, 11 (2016) 552-558. 